\title{
Risk and prognostic factors of post-catheterization pseudoaneurysm
}

\author{
Kenkichi Michimoto ${ }^{1 A, C, E}$, Shinsuke Takenaga ${ }^{1 B, D}$, Yo Matsui ${ }^{1 B, D}$, Keitaro Enoki ${ }^{1 F}$, Yosuke Nozawa ${ }^{1 F}$, \\ Takahiro Higuchi ${ }^{1 B, D}$, Hiroshi Sakamoto ${ }^{2 B}$, Yasuto Noda ${ }^{3 B}$, Satoru Morooka ${ }^{3 B}$ \\ 'Department of Radiology, Fuji City General Hospital, Japan \\ 2Department of Cardiology, Fuji City General Hospital, Japan \\ ${ }^{3}$ Department of Neurosurgery, Fuji City General Hospital, Japan
}

\section{Abstract}

Purpose: To evaluate the risk and prognostic factors of post-catheterization pseudoaneurysm (PPA).

Material and methods: To identify the risk factors for PPA occurrence, clinical findings were compared between 22 consecutive patients with radiologically confirmed PPAs (PPA group) and 300 randomly extracted patients without PPA, who underwent transarterial angiography or intervention (sample group) between 1 January 2015 and 31 March 2020. The PPA group was further divided into those treated successfully with mechanical compression (group A) and those requiring ultrasound-guided thrombin injection after compression failed (group B). Univariate and multivariate analyses were used to compare patient demographics, preoperative laboratory findings, procedure details, PPA diameter, and time interval between the procedure and compression between groups A and B to evaluate the prognostic factors of PPA.

Results: The PPA group demonstrated significantly elevated prothrombin time international normalized ratios (PT/INR) (odds ratio [OR]: 6.27, 95\% confidence interval [CI]: 2.020-19.5; $p=0.00151$ ) and more frequent popliteal access (OR: 14.2, 95\% CI: 1.040-195.0; $p=0.0467$ ) compared to the sample group, and radial access decreased the risk of PPA (OR: 0.382, 95\% CI: 0.0148-0.987; $p=0.0468$ ). One of the 22 PPAs resolved spontaneously, and 11 others (52.4\%) were successfully treated by mechanical compression. An interval exceeding 24 hours between the procedure and compression was the only significant prognostic factor $(p=0.0281)$ between groups A and B.

Conclusions: Elevated PT/INR and popliteal access may predispose patients to PPA; close consideration of the site of access may lower the risk of refractory PPA.

Key words: catheterization, prognostic factor, pseudoaneurysm, risk factor.

\section{Introduction}

Post-catheterization pseudoaneurysm (PPA) is an extremely rare complication that arises at the site of access after various diagnostic and interventional angiography procedures. Its reported incidence is $0.03-0.2 \%$ with radial access [1-3] and $0.3-0.7 \%$ with femoral access [2,3]. A pseudoaneurysm is a locally contained haematoma with arterial blood flow, which lacks a fibrous arterial wall and is surrounded by soft tissue. Clinical findings may include a pulsatile mass, localized pain, swelling, and expanding haematoma. Mechanical compression with ultrasound guidance is the first-line treatment to repair PPAs because the procedure is safe and cost-effective [4]. For intractable cases, thrombin injection (TI) or surgical repair can be alternatives. Reported risk factors for the formation of a PPA include patient age above 75 years, female gender, presence of infection, atrial fibrillation, hypertension, chronic kidney disease (CKD),

Correspondence address:

Dr. Kenkichi Michimoto, Department of Radiology, Fuji City General Hospital, Japan, e-mail: kenkichi.m@gmail.com

Authors' contribution:

A Study design · B Data collection · C Statistical analysis · D Data interpretation - E Manuscript preparation · F Literature search · G Funds collection 
intraoperative use of anticoagulants, brachial rather than femoral approach to catheterization, multiple puncture attempts, inadequate post-procedural haemostasis, and large sheath size [5-10]. The use of anticoagulants can complicate compression repair for PPA [11]. However, there is disagreement about these risks and the prognostic factors of PPA. To evaluate the risk of PPA development and associated prognostic factors, we investigated cross-sectoral catheterizations performed within our cardiology, cerebrovascular, and radiology departments.

\section{Material and methods}

\section{Study design}

Our institutional review board approved this single-centre retrospective analysis. From 1 January 2015 through 31 March 2020, 6401 transcatheter angiography procedures and/or interventions with arterial access were performed at our institution. These included coronary angiography (CAG), percutaneous coronary intervention (PCI), cerebral angiography, cerebral aneurysm embolization, angioplasty with/without stenting for peripheral arterial disease (PAD), embolization procedures including transcatheter arterial chemoembolization for hepatocellular carcinoma, uterine fibroid embolization, bronchial artery embolization for haemoptysis, and transcatheter arterial embolization for arterial haemorrhage. Of these, 22 consecutive PPAs in the same number of patients with radiological confirmation by ultrasound or computed tomography (CT) angiography were identified (PPA group). To identify the risk factors for the development of PPA, we compared the baseline characteristics of the PPA group that were thought to be associated with the incidence of PPA with those of a sample group of 300 randomly extracted patients without PPA who underwent transcatheter angiography procedures or interventions during the same period. The PPA group was further divided into those successfully treated with mechanical compression (group A) and those who required ultrasound-guided TI after failed mechanical compression (group B), and groups $\mathrm{A}$ and $\mathrm{B}$ were compared to determine the prognostic factors of PPA.

\section{Angiography procedure}

Details of angiography procedures, such as access site, size of the inserted sheath, amount of intraoperative administration of anticoagulant, and utilization of closure device for the access site, varied depending on the procedures and departments. For CAG and most conventional PCIs, right radial access was the first choice. Other access sites, including the left radial, brachial, and femoral arteries, were chosen in cases that required vascular access for haemodialysis in the right upper limb, occlusion of the right radial artery, and insertion of a large sheath. For procedures other than CAG and PCI, femoral access was the first choice; very rarely, popliteal access was chosen for endovascular treatment of PAD. In cases with radial access, after the procedure was completed, a compression band (TR BAND ${ }^{\circledR}$; Terumo Corporation, Tokyo, Japan) was placed over the access site and left in place for 6 hours. In cases with femoral access, AngioSeal (Terumo) was occasionally employed at the discretion of the surgeon. Patients generally received intraoperative heparin administration, consisting of 2000 units for CAG and cerebral artery angiography and 5000 units for PCI, PAD, and cerebral aneurysm embolization.

\section{Management for PPA}

All PPAs were first managed by manual compression with or without ultrasound assistance and additional continuous compression using a TR BAND ${ }^{\circledR}$ or elastic bandage until ultrasound confirmation the next day. For intractable cases, manual compression was repeated or TI was attempted. TI was performed by positioning a 23-gauge needle in the sac or neck of the PPA under ultrasound guidance and injecting $500 \mathrm{IU} / \mathrm{ml}$ of diluted thrombin (Fuji Pharma Co., Ltd., Tokyo, Japan) in a stepwise manner utilizing a 1-mL syringe under ultrasound monitoring. Treatment success was evaluated with ultrasonography and defined as the disappearance of the vascular signal into the sac of the PPA. All procedures were performed without conscious sedation. In patients with intolerable pain at the access site, local anaesthesia using $1 \%$ xylocaine was administered. All these procedures were performed after acquisition of the patient's written informed consent.

\section{Data collection and management}

We collected data regarding patient demographics including age, gender, body mass index (BMI), background disease for transcatheter arterial angiography or intervention, and past medical history or treatment of hypertension (HT), dyslipidaemia (DL), diabetes mellitus (DM), chronic kidney disease (CKD; defined as an estimated glomerular filtration rate (eGFR) below $60 \mathrm{ml} / \mathrm{min} / 1.73$ $\mathrm{m}^{2}$ ), haemodialysis, and atrial fibrillation (Af). Preoperative laboratory findings, including platelet count, prothrombin time international normalized ratio (PT/ INR), activated partial thromboplastin time (APTT), and fibrinogen levels, were compared between the groups. We also collected details regarding perioperative medications, such as single antiplatelet therapy (SAPT), dual antiplatelet therapy (DAPT), and anticoagulant administration, as well as procedure details, including access site (radial, brachial, femoral, and popliteal arteries), inserted sheath size, and intraoperative heparin administration. Days of hospitalization and period of follow-up after the procedure were also compared between the PPA and sample 
groups. For patients with PPAs, the diameter of the aneurysmal sac and neck, the time interval from the procedure to compression of the PPA (categorized into 0-24 and over 24 hours), and the number of trials of mechanical compression were compared between groups $\mathrm{A}$ and $\mathrm{B}$ to determine the prognostic factors of PPA.

\section{Statistical analysis}

Categorical variables are presented as number of cases (percentage) and compared between the groups using Fisher's exact test. Continuous variables are expressed as the mean \pm standard deviation (SD). For each continuous variable, we evaluated histograms and/or results of the Kolmogorov-Smirnov test to determine whether the continuous variables were normally distributed. We used the unpaired Student's $t$-test to compare the continuous variables with normal distribution between the groups and the Mann-Whitney $U$ test to compare the other continuous variables. Multivariable logistic regression analysis was used to identify the independent risk factors of PPA. A p-value below 0.05 was considered statistically significant. All data analyses were performed using EZR statistical software (Saitama Medical Centre, Jichi Medical University, Saitama, Japan; http://www.jichi.ac.jp/saitamasct/SaitamaHP.files/statmedEN.html) [12].

\section{Results}

A total of 22 PPAs were identified during the study period. Table 1 summarizes the baseline demographics, laboratory values, and procedure details of the PPA and sample groups. The sample group underwent significantly more CAG and PCI procedures and less angioplasty or stenting for PAD, and the PPA group demonstrated significantly greater PT/INRs, more cases with popliteal access, and significantly fewer cases with radial access. Other characteristics, including age, gender, BMI, HT, DL, DM, CKD, HD, Af, platelet count, APTT, fibrinogen, antiplatelet therapy, SAPT, DAPT, anticoagulant medication, and inserted sheath size, were not significant predictors of PPA. The PPA group also experienced significantly longer hospitalizations. Multivariable analysis demonstrated significant association between PT/INR above $1.5(p=0.00151)$, radial access $(p=0.0468)$, and popliteal access $(p=0.0467)$ and the occurrence of PPA (Table 2).

One of the 22 PPAs thrombosed spontaneously, 11 were treated with mechanical compression (group A); and ten required TI after failed attempts at mechanical compression (group B). Table 3 details the demographics of groups A and B. The only significant prognostic factor of PPA ( $p=0.0281$ ) was the time interval between the end of the procedure and initiation of compression. Other demographics, laboratory values, procedure details, sizes of PPAs, utilization of ultrasound guidance for compression, number of attempts at compression, and length of time for compression in each session were equal between groups $A$ and B. In group B, single sessions of TI were successfully performed using an average dose of $525 \pm 368$ IU (range, 200-1500 IU) of thrombin. Outpatient clinical follow-up for the PPA group revealed no recanalization or complications at an average follow-up of 12.7 months (range, 1-41 months).

\section{Discussion}

In this investigation, the incidence of PPA was significantly lower in patients who underwent CAG or PCI and radial access and significantly higher in cases with greater PT/INR and popliteal access. In addition, detection of PPA more than 24 hours after the procedure could complicate mechanical compression of PPA.

In our institution, most CAG and PCI procedures are performed using radial access. The radial route has been reported to reduce access-site complications including PPA in coronary and non-coronary interventions [13,14], and our study also demonstrated a lower risk of PPA development in a radial approach. The lower incidence of PPAs in patients undergoing CAG or PCI is thought to be attributable to the more frequent choice of radial access in those procedures. On the other hand, a significantly higher incidence of PPAs was observed in popliteal access. Pseudoaneurysm of the popliteal artery is more commonly reported following a surgical operation such as total knee arthroplasty or trauma [15], and little is reported about the development of pseudoaneurysms following popliteal artery access for catheterization. Although our sample size is too small to derive any conclusions, this comparatively infrequent access site may increase the risk of PPA formation.

PT/INR is a widely accepted index to evaluate clotting time upon activation of the extrinsic and common coagulation pathway and to monitor the effectiveness of anticoagulation therapy. PT/INR is elevated in patients such as those receiving warfarin therapy or lupus anticoagulants or with liver dysfunction, bile duct obstruction, vitamin K deficiency, coagulation factor deficiencies, and disseminated intravascular coagulation. Indeed, our study suggested an association of increased risk of PPA with elevated PT/INR, which was caused by anticoagulant medications as well as the other conditions mentioned above. The presence of anticoagulant medications including warfarin therapy and heparinization, intraoperative administration of heparin, and APTT were not significant predictors of PPA. A previous report suggested that PT/INR is a more reliable indicator of coagulopathy than APTT [16], and so PT/INR can be the most noteworthy parameter as a predictor of PPAs. This result supports the consensus guidelines for periprocedural management of percutaneous imageguided interventions [13], which recommend correction 
Table 1. Comparison of clinical characteristics between the post-catheterization pseudoaneurysm and sample groups

\begin{tabular}{|c|c|c|c|}
\hline Variable & $\begin{array}{l}\text { PPA group } \\
(n=22)\end{array}$ & $\begin{array}{l}\text { Sample group } \\
\qquad(n=300)\end{array}$ & $p$-value \\
\hline Age (years), mean $\pm S D$ & $70.5 \pm 11.9$ & $68.2 \pm 11.6$ & $0.363^{* *}$ \\
\hline Male gender, $n(\%)$ & $10(45.5)$ & $198(66.0)$ & $0.065^{*}$ \\
\hline Body mass index $\left(\mathrm{kg} / \mathrm{m}^{2}\right)$, mean $\pm \mathrm{SD}$ & $21.9 \pm 3.2$ & $23.6 \pm 4.0$ & $0.0547^{* *}$ \\
\hline Hypertension, $n(\%)$ & $20(90.9)$ & $240(80.0)$ & $0.272^{*}$ \\
\hline Dyslipidaemia, $n$ (\%) & $14(63.6)$ & $202(67.3)$ & $0.815^{*}$ \\
\hline Diabetes mellitus, $n(\%)$ & $5(22.7)$ & $80(26.7)$ & $0.806^{*}$ \\
\hline Chronic kidney disease, $n(\%)$ & $11(50)$ & $114(38.0)$ & $0.268^{*}$ \\
\hline Haemodialysis, $n(\%)$ & $1(4.5)$ & $25(8.3)$ & $1^{*}$ \\
\hline Atrial fibrillation, $n(\%)$ & $2(9.1)$ & $18(6.0)$ & $0.637^{*}$ \\
\hline Platelet count $\left(10^{3} / \mu \mathrm{l}\right)$, mean $\pm \mathrm{SD}$ & $216 \pm 70$ & $227 \pm 78$ & $0.503^{* *}$ \\
\hline $\mathrm{PT} /$ INR, mean $\pm \mathrm{SD}$ & $1.38 \pm 0.91$ & $1.07 \pm 0.34$ & $0.0106^{* * *}$ \\
\hline PT/INR > $1.5(\%)$ & $6(27.3)$ & $16(5.3)$ & $0.00213^{* * *}$ \\
\hline APTT $(s)$, mean $\pm S D$ & $43.4 \pm 46.8$ & $41.0 \pm 54.0$ & $0.841^{* *}$ \\
\hline Fibrinogen $(\mathrm{mg} / \mathrm{dl})$, mean $\pm S D$ & $334 \pm 76$ & $350 \pm 87$ & $0.468^{* *}$ \\
\hline \multicolumn{4}{|l|}{ Medication, $n(\%)$} \\
\hline Single antiplatelet therapy & $3(13.6)$ & $55(18.3)$ & $0.776^{*}$ \\
\hline Dual antiplatelet therapy & $15(68.2)$ & $179(59.7)$ & $0.504^{*}$ \\
\hline Anticoagulative therapy & $8(36.3)$ & $60(20.0)$ & $0.0996^{*}$ \\
\hline \multicolumn{4}{|l|}{ Access site, $n(\%)$} \\
\hline Radial & $8(36.4)$ & $186(62.0)$ & $0.0233^{*}$ \\
\hline Brachial & $2(9.1)$ & $21(7.0)$ & $0.664^{*}$ \\
\hline Femoral & $10(45.5)$ & $91(30.3)$ & $0.156^{*}$ \\
\hline Popliteal & $2(9.1)$ & $2(0.7)$ & $0.0246^{*}$ \\
\hline Sheath size (French), mean \pm SD & $5.23 \pm 1.57$ & $5.55 \pm 0.78$ & $0.225^{* * *}$ \\
\hline Intraoperative heparin dose (units), mean \pm SD & $3,050 \pm 2,130$ & $2,560 \pm 1,800$ & $0.28^{* * *}$ \\
\hline Hospitalization period (days), mean $\pm S D$ & $26.0 \pm 29.1$ & $9.37 \pm 12.2$ & $<0.01^{* *}$ \\
\hline Follow-up period (months), mean \pm SD & $12.7 \pm 12.9$ & $8.91 \pm 10.7$ & $0.116^{* *}$ \\
\hline \multicolumn{4}{|l|}{ Background, $n(\%)$} \\
\hline CAG/elective PCI & $8(36.4)$ & $198(66.0)$ & $0.00968^{*}$ \\
\hline Emergency PCl & $4(18.2)$ & $45(15.0)$ & $0.757^{*}$ \\
\hline Cerebral embolization & $3(13.6)$ & $21(7.0)$ & $0.219^{*}$ \\
\hline Angioplasty or stenting for PAD & $4(18.2)$ & $15(5.0)$ & $0.0321^{*}$ \\
\hline Others & $3(13.6)$ & $21(7.0)$ & $0.219^{*}$ \\
\hline
\end{tabular}

APTT - activated partial thromboplastin time, CAG - coronary angiography, PAD - peripheral arterial disease, PCI - percutaneous coronary intervention, PPA - post-catheterization pseudoaneurysm, PT/INR - prothrombin time international normalized ratio, SD - standard deviation.

*Denotes p-values obtained using Fisher's exact test. **Denotes p-values obtained using Student's $t$-test. ***Denotes $p$-values obtained using Mann-Whitney U test

Table 2. Multivariate logistic regression analysis to assess the risk factors of post-catheterization pseudoaneurysm

\begin{tabular}{|l|c|c|c|}
\hline Variable & Odds ratio & $95 \% \mathrm{Cl}$ & $p$-value \\
\hline PT/INR $>1.5$ & 6.27 & $2.020-19.500$ & 0.0015 \\
\hline Radial access & 0.38 & $0.015-0.987$ & 0.0468 \\
\hline Popliteal access & 14.20 & $1.040-195.000$ & 0.0467 \\
\hline PT/INR - prothrombin time international normalized ratio & \\
\hline
\end{tabular}


Table 3. Comparison of the clinical characteristics between groups $A$ and $B$

\begin{tabular}{|c|c|c|c|}
\hline Variable & $\begin{array}{l}\text { Group A } \\
(n=11)\end{array}$ & $\begin{array}{l}\text { Group B } \\
(n=10)\end{array}$ & $p$-value \\
\hline Age (years), mean $\pm S D$ & $75.5 \pm 9.2$ & $66.6 \pm 12.6$ & $0.0776^{* *}$ \\
\hline Male gender, $n(\%)$ & $4(36.4)$ & $5(50.0)$ & $0.67^{*}$ \\
\hline Body mass index $\left(\mathrm{kg} / \mathrm{m}^{2}\right)$, mean $\pm S D$ & $21.7 \pm 3.0$ & $21.4 \pm 2.5$ & $0.773^{* *}$ \\
\hline Hypertension, $n(\%)$ & $10(90.9)$ & $9(90.0)$ & $1^{*}$ \\
\hline Dyslipidaemia, $n(\%)$ & $8(72.7)$ & $5(50.0)$ & $0.387^{*}$ \\
\hline Diabetes mellitus, $n(\%)$ & $3(27.3)$ & $2(20.0)$ & $1^{*}$ \\
\hline Chronic kidney disease, $n(\%)$ & $7(63.6)$ & $4(40.0)$ & $0.395^{*}$ \\
\hline Haemodialysis, $n(\%)$ & $1(9.1)$ & $0(0)$ & $1^{*}$ \\
\hline Atrial fibrillation, $n(\%)$ & $1(9.1)$ & $1(10.0)$ & $1^{*}$ \\
\hline Platelet count $\left(10^{3} / \mu \mathrm{l}\right)$, mean $\pm S D$ & $191 \pm 49$ & $247 \pm 81$ & $0.0661^{* *}$ \\
\hline PT/INR, mean \pm SD & $1.40 \pm 1.14$ & $1.39 \pm 0.69$ & $0.974^{* * *}$ \\
\hline PT/INR > $1.5(\%)$ & $2(18.2)$ & $4(40.0)$ & $0.361^{* * *}$ \\
\hline APTT (s), mean $\pm S D$ & $51.6 \pm 65.9$ & $34.9 \pm 10.5$ & $0.438^{* *}$ \\
\hline Fibrinogen (mg/dl), mean \pm SD & $325 \pm 88$ & $334 \pm 14$ & $0.854^{* *}$ \\
\hline \multicolumn{4}{|l|}{ Medication, $n(\%)$} \\
\hline Single antiplatelet therapy & $0(0)$ & $2(20.0)$ & $0.214^{*}$ \\
\hline Dual antiplatelet therapy & $10(81.8)$ & $5(50.0)$ & $0.0635^{*}$ \\
\hline Anticoagulative therapy & $2(18.2)$ & $6(60.0)$ & $0.0805^{*}$ \\
\hline \multicolumn{4}{|l|}{ Access site, $n(\%)$} \\
\hline Radial & $2(18.2)$ & $5(50.0)$ & $0.183^{*}$ \\
\hline Brachial & $1(90.9)$ & $1(10.0)$ & $1^{*}$ \\
\hline Femoral & $6(54.5)$ & $4(40.0)$ & $0.67^{*}$ \\
\hline Popliteal & $2(18.2)$ & $0(0)$ & $0.476^{*}$ \\
\hline Sheath size (French), mean \pm SD & $5.45 \pm 1.57$ & $4.90 \pm 1.66$ & $0.43^{* * *}$ \\
\hline Intraoperative heparin dose (units), mean \pm SD & $3.54 \pm 2.11$ & $2.30 \pm 20.6$ & $0.196^{* * *}$ \\
\hline Hospitalization period (days), mean \pm SD & $27.4 \pm 22.0$ & $26.6 \pm 37.1$ & $0.966^{* *}$ \\
\hline Follow up period (months), mean $\pm S D$ & $12.4 \pm 11.8$ & $10.6 \pm 12.7$ & $0.745^{* *}$ \\
\hline Sac diameter of aneurysm $(\mathrm{mm})$, mean \pm SD & $13.2 \pm 6.4$ & $16.2 \pm 16.7$ & $0.635^{* *}$ \\
\hline Neck length of aneurysm $(\mathrm{mm})$, mean \pm SD & $2.4 \pm 1.0$ & $1.9 \pm 1.0$ & $0.323^{* * *}$ \\
\hline \multicolumn{4}{|l|}{ Time interval between the procedure and compression } \\
\hline $0-24$ hours & $7(63.6)$ & $1(10.0)$ & \multirow[t]{2}{*}{$0.0281^{* *}$} \\
\hline Over 24 hours & $4(36.4)$ & $9(90.0)$ & \\
\hline Utilization of ultrasound guidance for compression & $7(63.6)$ & $7(70.0)$ & $1^{*}$ \\
\hline Number of attempts of compression & $1.1 \pm 0.3$ & $1.6 \pm 0.8$ & $0.0982^{* * *}$ \\
\hline Duration of compression in each session, minutes & $36.9 \pm 15.6$ & $31.9 \pm 27.3$ & $0.203^{* *}$ \\
\hline
\end{tabular}

APTT - activated partial thromboplastin time, CAG - coronary angiography, PAD - peripheral arterial disease, PCI - percutaneous coronary intervention, PPA - post-catheterization pseudoaneurysm, PT/INR - prothrombin time international normalized ratio, SD - standard deviation.

*Denotes $p$-values obtained using Fisher's exact test. **Denotes $p$-values obtained using Student's $t$-test. ***Denotes $p$-values obtained using Mann-Whitney $U$ test

of the PT/INR to $<1.5$ for procedures with moderate bleeding risk, such as angiography and arterial embolization.

In this study, no significant difference between the PPA and sample groups was observed regarding other previously reported predisposing factors for PPA, such as older age, female gender, medical history of HT, CKD, or Af, sheath size used in the procedure, and use of brachial access [5-10]. Further comprehensive investigation is desirable to determine the risk factors of PPA. 
The reported $72-93 \%$ success rate of mechanical compression can fall in cases with anticoagulation status, PPA size exceeding $2 \mathrm{~cm}$, and obesity [11,17-19]. Generally, the use of compression to treat PPA is easy, cost-effective, and safe and yields an acceptable success rate, but it is timeconsuming and sometimes requires intravenous sedation because of pain during, and at the site of, compression [4]. Our finding of relatively lower success with mechanical compression for PPA (11 of 21 PPAs [52.4\%]) may be attributable to the absence from our compression protocol of a standard guideline regarding the length of time of compression, whether and how to employ ultrasonography and intravenous sedation, and when to switch to TI. We found that detection of PPA more than 24 hours after the procedure can complicate repair by mechanical compression, and successful repair has been associated with the time interval between catheter removal and compression [11]. Thus, prompt recovery from PPA requires close and continuous evaluation of the access site after the procedure.

Although thrombin is still unlicensed for the treatment of PPA, TI has been proven as a safe and reliable procedure for PPA, with a reported success rate of $86-98 \%$ by a directly made thrombus followed by convertion of inactive fibrinogen into fibrin $[4,6,20]$. TI is feasible even for PPAs after failed attempts with mechanical compression [2]. The injection of a diluted thrombin to 100 or $1000 \mathrm{IU} / \mathrm{ml}$ has been reported [4], but the optimal concentration remains unclear. Injection of a lower concentration of thrombin may increase the amount of thrombin, and use of a higher concentration may increase the risk of nontargeted thrombus. In this study, thrombin diluted to $500 \mathrm{IU} / \mathrm{ml}$ was employed and demonstrated satisfactory efficacy and safety. Potential risks of TI are thrombosis in the native or distal arteries, allergic reactions to thrombin, and recanalization [6]. Although surgical repair has been the gold standard to treat PPA, it requires the use of general anaesthesia and is more expensive than TI. Hence, thrombin injection is a promising alternative for intractable PPA.

Our study is limited by its retrospective nature, singlecentre design, relatively small population with PPAs, and no consideration about the difficulty and adequacy of the procedures involved, including puncture and haemostasis at the site of access.

In summary, the risk of PPA can increase in cases in which the PT/INR exceeds 1.5 and popliteal access is used, and the risk decreases with radial access. In addition, the detection of PPA more than 24 hours after the procedure can be a prognostic factor of ineffective mechanical compression of the PPA.

\section{Conflicts of interest}

The authors declare that they have no conflicts of interest.

\section{References}

1. Jolly SS, Yusuf S, Cairns J, et al. Radial versus femoral access for coronary angiography and intervention in patients with acute coronary syndromes (RIVAL): a randomised, parallel group, multicentre trial. Lancet 2011; 377: 1409-1420.

2. Zegrí I, García-Touchard A, Cuenca S, et al. Radial artery pseudoaneurysm following cardiac catheterization: clinical features and nonsurgical treatment results. Rev Esp Cardiol (Engl Ed) 2015; 68: 349-351.

3. Scheer B, Perel A, Pfeiffer UJ. Clinical review: complications and risk factors of peripheral arterial catheters used for haemodynamic monitoring in anaesthesia and intensive care medicine. Crit Care 2002; 6: 199-204.

4. Sarioglu O, Capar AE, Belet U. Interventional treatment options in pseudoaneurysms: different techniques in different localizations. Pol J Radiol 2019; 84: e319-e327.

5. Swanson E, Freiberg A, Salter DR. Radial artery infections and aneurysms after catheterization. J Hand Surg Am 1990; 15: 166-171.

6. Garvin RP, Ryer EJ, Yoon HR, et al. Ultrasound-guided percutaneous thrombin injection of iatrogenic upper extremity pseudoaneurysms. J Vasc Surg 2014; 59: 1664-1669.

7. Collins N, Wainstein R, Ward M, et al. Pseudoaneurysm after transradial cardiac catheterization: case series and review of the literature. Catheter Cardiovasc Interv 2012; 80: 283-287.
8. Otsuka M, Shiode N, Nakao Y, et al. Comparison of radial, brachial, and femoral accesses using hemostatic devices for percutaneous coronary intervention. Cardiovasc Interv Ther 2018; 33: 62-69.

9. Ayhan E, Isik T, Uyarel H, et al. Femoral pseudoaneurysm in patients undergoing primary percutaneous coronary intervention for ST-elevation myocardial infarction: incidence, clinical course and risk factors. Int Angiol 2012; 31: 579-585.

10. Tamanaha Y, Sakakura K, Taniguchi Y, et al. Comparison of postcatheterization pseudoaneurysm between brachial access and femoral access. Int Heart J 2019; 60: 1030-1036.

11. Eisenberg L, Paulson EK, Kliewer MA, et al. Sonographically guided compression repair of pseudoaneurysms: further experience from a single institution. AJR Am J Roentgenol 1999; 173: 1567-1573.

12. Kanda Y. Investigation of the freely available easy-to-use software 'EZR' for medical statistics. Bone Marrow Transplant 2013; 48: 452-458.

13. Patel IJ, Davidson JC, Nikolic B, et al. Consensus guidelines for periprocedural management of coagulation status and hemostasis risk in percutaneous image-guided interventions. J Vasc Interv Radiol 2012; 23: 727-736.

14. Eichhofer J, Horlick E, Ivanov J, et al. Decreased complication rates using the transradial compared to the transfemoral approach in percutaneous coronary intervention in the era of routine stenting and 
glycoprotein platelet IIb/IIIa inhibitor use: a large single-center experience. Am Heart J 2008; 156: 864-870.

15. Ammori MB, Evans AR, Mclain AD. Popliteal artery pseudoaneurysm after total knee arthroplasty. J Arthroplasty 2016; 31: 2004 2007.

16. Yuan S, Ferrell C, Chandler WL. Comparing the prothrombin time INR versus the APTT to evaluate the coagulopathy of acute trauma. Thromb Res 2007; 120: 29-37.

17. Coley BD, Roberts AC, Fellmeth BD, et al. Postangiographic femoral artery pseudoaneurysms: further experience with US-guided compression repair. Radiology 1995; 194: 307-311.
18. Schaub F, Theiss W, Busch R, et al. Management of 219 consecutive cases of postcatheterization pseudoaneurysm. J Am Coll Cardiol 1997; 30: 670-675.

19. Morgan R, Belli AM. Current treatment methods for postcatheterization pseudoaneurysms. J Vasc Interv Radiol 2003; 14: 697-710.

20. Jargiełło T, Sobstyl J, Światłowski Ł, et al. Ultrasound-guided thrombin injection in the management of pseudoaneurysm after percutaneous arterial access. J Ultrason 2018; 18: 85-89. 